\title{
Dynamic Multicompartment Refrigerated Vehicle Routing Problem with Multigraph Based on Real-Time Traffic Information
}

\author{
Deng-Kai Hou $\mathbb{D}^{\mathbb{D}},{ }^{1}$ Hou-Ming Fan $\mathbb{D}^{1},{ }^{1,2}$ Ying-Chun Lv $\mathbb{D}^{1,2}$ and Xiao-Xue Ren ${ }^{1,2}$ \\ ${ }^{1}$ College of Transportation Engineering, Dalian Maritime University, Dalian 116026, China \\ ${ }^{2}$ Institute of Strategy Management and System Planning, Dalian 116026, China \\ Correspondence should be addressed to Hou-Ming Fan; fhm468@dlmu.edu.cn
}

Received 23 January 2021; Revised 15 July 2021; Accepted 6 January 2022; Published 1 February 2022

Academic Editor: Hocine Imine

Copyright ( $\odot 2022$ Deng-Kai Hou et al. This is an open access article distributed under the Creative Commons Attribution License, which permits unrestricted use, distribution, and reproduction in any medium, provided the original work is properly cited.

Aiming at the dynamic multicompartment refrigerated vehicle routing problem with multigraph based on real-time traffic information, this study, based on the idea of preoptimization followed by real-time adjustment, establishes a two-stage mathematical model with minimizing delivery cost. In the preoptimization phase, this study, based on historical traffic information, designed a hybrid chaotic genetic algorithm with variable neighborhood search (HCGAVNS) to obtain the initial delivery scheme. In the real-time adjustment phase, the order in which customers are served remains the same and a path selection strategy is proposed to solve the problem according to the real-time traffic information of different paths. The validity of the model and the algorithm are verified through the analysis of instances. The research results can enrich the related research on cold chain vehicle routing problem and provide a theoretical basis for logistics companies to optimize their delivery scheme.

\section{Introduction}

Dynamic multicompartment refrigerated vehicle routing problem with multigraph based on real-time traffic information is a dynamic vehicle routing problem (DVRP) based on real-time traffic information provided by traffic big data, considering the multigraph between two nodes and realtime adjustment of the driving route. With the continuous development of the e-commerce network, customers' requirements for timeliness and quality of fresh products are constantly improving. Logistics enterprises are required to deliver fresh products to customers as quickly as possible. However, the driving speed of vehicles is affected by realtime traffic conditions. There are some paths between two nodes in the delivery network. The different paths have different real-time traffic information. Depots need to plan the delivery routes according to the actual road conditions and allocate appropriate paths to reduce the cargo damage cost and improve delivery timeliness. Multicompartment refrigerated vehicles have been widely used in recent years because they can meet customers' requirements for different fresh-keeping temperatures. For example, supermarkets may have delivery requirements for fresh products such as seafood, raw meat, and vegetables at the same time. If a single compartment refrigerated vehicle is used, multiple deliveries are required. While a multicompartment refrigerated vehicle can meet the delivery requirements at one time, many scholars have studied the DVRP based on realtime traffic information, DVRP with multigraph, and multicompartment refrigerated VRP.

For DVRP based on real-time traffic information, Tang et al. [1] considered the relationship between speed and time in a delivery network and designed a multiobjective particle swarm optimization algorithm to solve the problem. Lin et al. [2] designed an improved A-star exact solution algorithm and a hybrid simulated annealing algorithm to solve the DVRP problem. Considering the time-dependent delivery network, Ge and Zhang [3] applied the improved genetic algorithm to solve the problem. Tong [4] applied a tabu search algorithm to solve VRP based on real-time information. Li et al. [5] considered two situations of frequent and occasional traffic congestion in the delivery network and solved the problem by combining the genetic algorithm with Dijkstra algorithm. Kok et al. [6] avoided 
congested road sections by finding alternative paths and combined the Dijkstra algorithm with heuristic algorithm to solve the problem. Sabar et al. [7] applied an adaptive evolutionary algorithm to solve the DVRP problem considering traffic congestion. With the rapid development of ITS, more and more scholars have integrated traffic big data into the research of DVRP. Xu and Guo [8] used a big data platform to obtain real-time traffic information and applied a genetic algorithm based on MapReduce in Hadoop, the big data platform, to solve the problem in parallel. Tang et al. [9] collected and processed initial data by big data calculation and proposed a vehicle routing algorithm based on the big data calculation model to solve the problem. Yao and $\mathrm{He}$ [10] combined traffic big data with a cold chain delivery management information system and applied an ant colony algorithm to solve the problem.

Most of the traditional DVRP only considered the situation of a single path between two nodes. However, the real delivery network is complicated, and there is often more than one path between two nodes; the research of DVRP with multigraph is essential. Garaix et al. [11] introduced VRP with multigraph for the first time and proposed many possibilities in the path selection of two nodes. Ticha et al. [12] proposed a DVRP with multigraph and time windows and designed an adaptive large neighborhood search algorithm to solve it. Wang et al. [13] considered alternative paths and applied a two-stage particle swarm optimization algorithm to solve the problem. Huang et al. [14] established a mixed integer programming model considering deterministic and stochastic traffic conditions and solved it with an improved Dijkstra algorithm. Setak et al. [15] applied a heuristic algorithm based on a tabu search algorithm to solve the VRP problem with multigraph between two nodes. Qian and Eglese [16] took the path and vehicle speed as decision variables and applied a tabu search algorithm based on a column generation algorithm to solve the time-dependent DVRP with multigraph. Li et al. [17] researched a delivery network with multigraph attributes and applied a doublecycle simulated annealing algorithm to solve the problem.

The multicompartment refrigerated VRP is a kind of problem combining multicompartment VRP and cold chain VRP. To solve the multicompartment refrigerated VRP, Chen et al. [18] aiming at minimizing the total cost, applied particle swarm optimization algorithm combined with neighborhood search strategy to solve the problem. Wang and $\mathrm{Li}$ [19] applied a hybrid fruit fly optimization algorithm to solve the multicompartment VRP to minimize the total transportation distance. Martins et al. [20] allocated the delivery period according to the customer demand within one week and used the adaptive large neighborhood search algorithm to solve the problem to minimize the total delivery cost. Eshtehadi et al. [21] aimed at minimizing the fixed and variable delivery costs and applied the improved adaptive large neighborhood search algorithm to solve the multicompartment VRP. Kaabachi et al. [22] aimed at minimizing the number of vehicles and the distance and combined the ant colony algorithm with a hybrid adaptive generalized variable neighborhood algorithm to solve the problem. Chen et al. [23] considered the oil consumption in the waiting time, aiming at minimizing the total delivery cost and applied the ALNS algorithm to solve the problem. Ostermeier and Hübner [24] considered a mixed delivery method of two different types of vehicles, respectively: single compartment and multicompartment. It applied the fast large-scale domain search algorithm to solve the problem. Hu et al. [25] considered the characteristics of multitemperature codelivery and the time window constraints of customers and applied the genetic algorithm to solve the problem. Henke et al. [26] took the waste glass classification and recycling problem as an example and applied the variable neighborhood search algorithm to solve the problem. Goodson [27] considered the random demand and applied a simulated annealing algorithm to solve it. Alinaghian and Shokouhi [28] applied a hybrid algorithm combined large-scale neighborhood search with variable neighborhood search to solve the problem. Coelho and Leandro [29] applied the branch and bound method to solve the multicompartment delivery problem in oil transportation. In response to the cold chain VRP, Osvald and Stirn [30] considered the impact of the perishability of fresh products on the cost in the cold chain delivery process and effectively reduced the loss of fresh products. Miao et al. [31] considered the cost of fresh product loss in the delivery process and applied an improved genetic algorithm to solve the problem. Zhang and $\mathrm{Xu}$ [32] integrated the refrigeration cost into the total delivery cost and solved it with an improved genetic algorithm. Du and $\mathrm{Li}$ [33] considered the customer satisfaction, fresh product loss, and oil consumption in the fresh products delivery process and applied the improved simulated annealing algorithm to solve the fresh products delivery problem. Du and Li $[33,34]$ considered the cost of fresh product loss and fuel consumption in the total cost and applied a hybrid ant colony algorithm to solve the problem. Tao et al. [35] considered the cost of fresh product loss and a carbon tax in the delivery process and applied the global artificial fish swarm algorithm to solve the problem. Zhao et al. [36] considered the economic cost and environmental cost in delivery process and applied an improved ant colony algorithm to solve the problem. $\mathrm{Xu}$ [37] considered time window constraints and applied a genetic algorithm to solve the problem.

With the rapid development of the cold chain industry, more and more attention has been paid to the quality of fresh products. Through literature review, it can be seen that the existing research still has the following deficiencies: (1) for DVRP, based on real-time information, the road state or vehicle speed between two nodes is the same or the update frequency was low, and it ignores the real-time change of traffic information, resulting in large deviation from the actual situation; (2) for DVRP with multigraph, most of the delivery routes are schemed according to historical data, and few of the paths in delivery routes are updated according to the real-time traffic data; (3) for multicompartment refrigerated VRP, most references assume that the vehicle speed is constant during the delivery process, ignoring the impact of real-time traffic information. So, the delivery scheme based on constant speed is unable to solve the practical problems. Moreover, although most references consider the different fresh products loss costs in different 
temperature compartments, they seldom consider the relationship between fresh product loss costs and the remaining fresh products in the compartments, resulting in the inaccurate calculation of fresh product loss costs.

The refrigerated vehicle routing problem proposed in this paper is to optimize the storage and transportation links of cold chain logistics by considering the influence of refrigeration cost and cargo damage cost on the route optimization. Fresh products delivery has the characteristics of "multiple varieties and small batches." Refrigerated and frozen cargos have different requirements on temperature and humidity. If multiple cargos are transported separately, the loading rate of vehicles will be reduced, and the transportation cost will be increased. If multiple cargos are transported together, the product freshness will be reduced. In the delivery process, multicompartment refrigerated vehicles can not only improve the loading rate but also ensure the freshness of products, reducing the cargo damage and meeting the diversified needs of customers.

In view of the above deficiencies, the dynamic multicompartment refrigerated vehicle routing problem with multigraph based on real-time traffic information is studied. The novelty of this paper is as follows: first, few papers have considered the refrigerated vehicle routing problem with multigraph. The delivery time of refrigerated vehicle routing problem is very important. The speed of vehicles is different in different paths; by selecting different paths, the transportation time can be shortened, and the efficiency can be improved. Second, as for the vehicle routing problem with multigraph, once the selected path is fixed, it will not change. However, in the actual delivery process, the traffic information of different paths is time-varying, so the selection of the path must be updated in real-time according to the traffic information. Third, the refrigerated vehicle routing problem should not only consider the transportation cost and fixed cost but also the refrigeration cost and cargo damage cost in the process of transportation. For refrigerated and frozen cargos transportation, customers generally have time requirements, so the time penalty costs should be included.

The contributions of this paper can be summarized as follows:

(i) Considering the multiple paths between two nodes and the real-time traffic information in different paths, as well as the cargo damage cost and refrigeration cost in the delivery process, a two-stage mixed integer programming model is established based on the idea of preoptimization followed by real-time adjustment to minimize the total cost.

(ii) A hybrid variable neighborhood chaotic genetic algorithm is designed to solve the model. Pseudorandomness of chaotic system is introduced into the algorithm to ensure the diversity of initial solutions, and an adaptive neighborhood search number strategy is introduced to balance the breadth and depth required by population evolution.

\section{Data Acquisition and Processing}

Tang et al. [9] show that traffic big data have the $4 \mathrm{~V}$ characteristics of volume, velocity, variety, and veracity. In this paper, the path selection of dynamic road network is based on a big data processing platform, and the specific framework is shown in Figure 1. Historical traffic information, depot information, customer information, and cost information are integrated into one system. Historical traffic data include the daily traffic volume and occupancy data. At the same time, the vehicle routes, vehicle location, and customer information are outputted by the dynamic VRP module and are inputted into the multisource module together with updated traffic information. Among them, the traffic data are collected by the Twin Cities Highway in the United States (https://www.d.umn.edu/tdrl/services.htm). Through the TravelTimeMetroTC software (https://www.d. umn.edu/ tkwon/TDRLSoftware/Download.html), the daily traffic volume and occupancy data are used to estimate the vehicle speed of each road.

\section{Problem and Mathematical Model}

3.1. Problem Description. The dynamic multicompartment refrigerated vehicle routing problem with multigraph based on real-time traffic information studied in this paper is descripted as follows: the delivery network has a complete directed graph $G=(V, E)$, where nodes set is $V=\{0\} \cup V_{0}, 0$ represents the depot, and $V_{0}=\{1,2,3, \cdots, n\}$ represents the customer set. $E=\{(i, j) \mid i, j \in V\}$ represents the edge set. This paper considers that there are multiple paths between two nodes, as shown in Figure 2. $m$ represents any path in the path set $M=\{1,2,3, \cdots, m, \cdots, \eta\}$ between two nodes, and the vehicle only chooses one of them to travel and $l_{i j m}$ represents the distance of path $m$ between node $i$ and node $j$. $k$ represents a vehicle and each vehicle is equipped with two compartments, namely, the refrigerated compartment and the frozen compartment. The capacity of each compartment is equal to $Q_{h} . H$ represents the product set, $h=1$ represents the refrigerated cargos, $h=2$ represents the frozen cargos, and the demand for each product at the customer $i$ is $d_{i h}$.

The service time window of the customer is $\left[E T_{i}, L T_{i}\right]$, in which $E T_{i}$ represents the earliest acceptable service time of the customer $i$ and $L T_{i}$ represents the latest acceptable service time of the customer $i$. $\left[E T_{i}, L T_{i}\right]$ is the time window when the customer allows the vehicle to serve. There is no time penalty cost only when the vehicle serves the customer within the $\left[E T_{i}, L T_{i}\right]$. The vehicle arriving earlier or later than the customers' time window $\left[E T_{i}, L T_{i}\right]$ will incur penalty cost, in which $c_{1}$ represents the waiting cost per unit time and $c_{2}$ represents the delay cost per unit time. The working time window of the depot is $\left[T_{s}, T_{f}\right]$, and it means that the depot only operates within this time window. Vehicles must leave the depot after $T_{s}$ and return to the depot before $T_{f}$. There is an additional constraint that $L T_{i}$ must be less than $T_{f}$; otherwise, it may result in the vehicle 


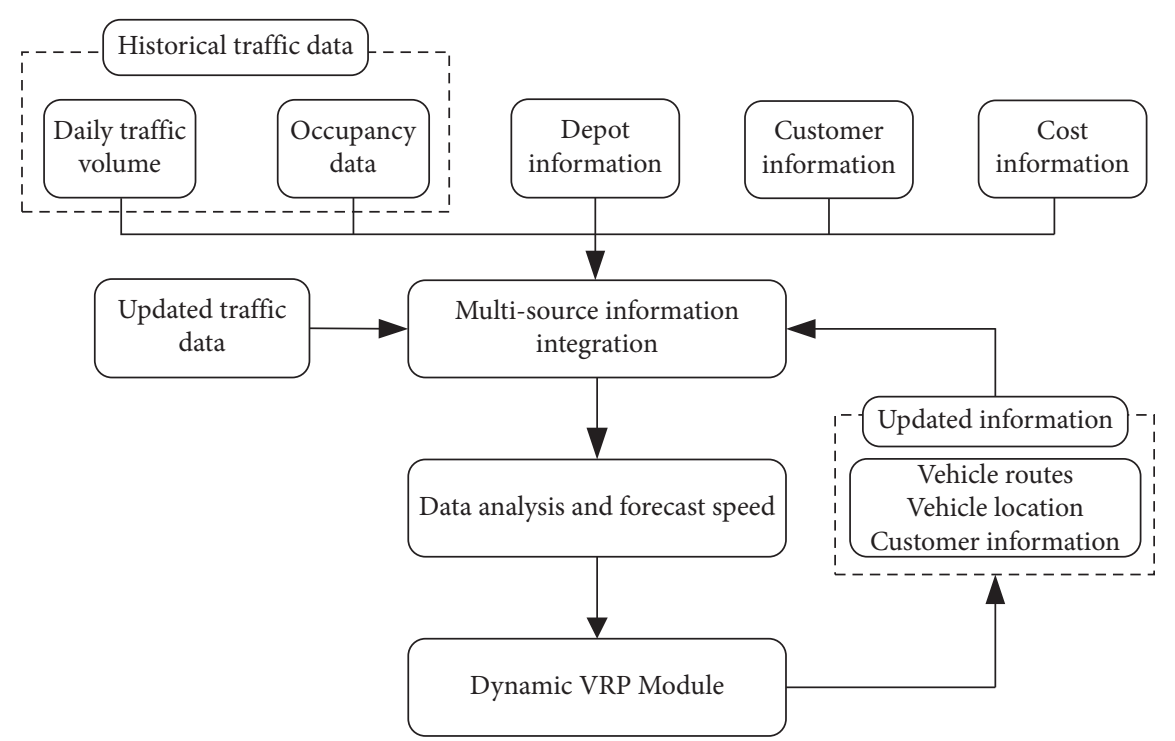

FIGURE 1: Information processing framework.

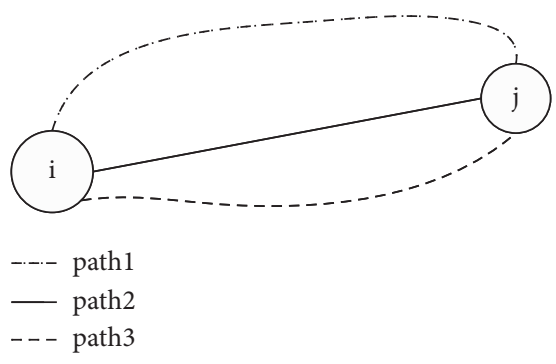

FIGURE 2: Schematic diagram of the path.

not returning to the depot before $T_{f} \cdot t_{i k}^{s}$ represents the service time at customer $i$. $c_{3}$ represents the unit fuel consumption cost; $c_{4}$ represents the vehicle fixed cost. The decision variable $x_{i j k m}$ indicates whether the vehicle $k$ goes from node $i$ to node $j$ through the path $m$, which is 1 and not 0 . The problem to be solved is that the depot preoptimizes the delivery route according to the known customer information, historical traffic information, and the customer demand for different kinds of fresh products. During the delivery process, the path selected may not be the optimal path as the delivery time and real-time traffic information change, so the path needs to be reselected according to the real-time traffic information to minimize the total delivery cost.

3.2. Speed Function Determination. In this paper, historical traffic information of roads is obtained through the big data platform, and a dynamic speed curve represents the predicted driving speed of a road section with an update frequency of 30 seconds per time. The update frequency is the same as real-time traffic information and the driving speed on a certain road section of a certain day is shown in Figure 3. Among them, the prediction of road speed is based on historical data. On weekdays, the speed data are predicted according to the historical data of the previous week displayed on the data platform. On the rest days, the speed data are predicted according to the historical data of rest days of the first five weeks displayed on the data platform.

First, the predicted traffic information on a day is divided into several periods at a time of 30 seconds, and the working hours of the depot are divided into $P$ periods according to the same frequency based on the delivery start time and traffic information. It is assumed that the time $T_{i k}$ when the vehicle departs from the node $i$ belongs to $\left[T_{p}, T_{p+1}\right]$. The predicted speed of the path is $v_{p m}$. There are two possibilities for vehicles traveling from node $i$ to node $j$, respectively: intraperiod and interperiod. Intraperiod refers to that the vehicle can arrive at node $j$ within the time period when it starts from node $i$, without crossing the time period. Interperiod refers to that the vehicle needs to cross multiple time periods to reach node $j$, and the speed will change once for each period. If $l_{i j m} \leq\left(T_{p+1}-T_{i k}\right) v_{p m}$, the vehicle arrives at the node $j$ within the first time period, there is no need to travel across time periods. Otherwise, it is necessary to travel across time periods. Assuming that the vehicle travels from node $i$ to node $j$ across $f$ time periods, the distance traveled in each period is $l_{i j m p}, l_{i j m(p+1)}, \ldots, l_{i j m(p+f)}$. Vehicle travels at speed $v_{p m}$ until it enters the $p+1$ time period. The speed of the vehicle in the $p+1$ time period is $v_{(p+1) m}$, and so on. The speed when the vehicle leaves the different paths of the road section $(i, j)$ is $v_{(p+f) m}$. At this time, the speed of the vehicle at different times of the different paths of the road section $(i, j)$ can be expressed as $v_{i j m}=\left\{v_{p m}, v_{(p+1) m}, \ldots \ldots v_{(p+f) m}\right\}$. In summary, the predicted travel time that a vehicle chooses a different path from node $i$ to node $j$ can be calculated by the following equation:

$$
t_{i j k m}=\left\{\begin{array}{l}
l_{i j m} / v_{p m}, \quad l_{i j m} \leq\left(T_{p}-T_{i k}\right) v_{p m}, \\
\sum_{f \in F} \frac{l_{i j m(p+f)}}{v_{m(p+f)}}, \quad \text { otherwise. }
\end{array}\right.
$$




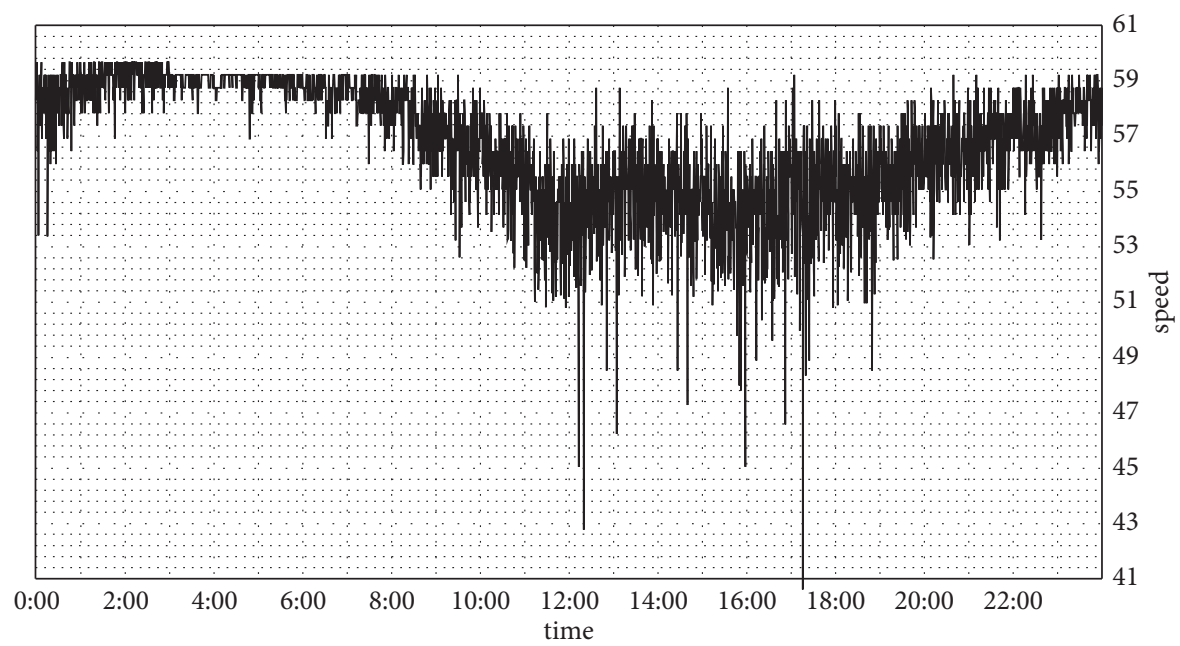

FiguRE 3: Driving speed on a certain road section of a day.

As shown in Figure 4, the time that the vehicle leaves node $i$ is the ending moment of time period $T_{2}$. There are two possibilities for the time of the vehicle arriving at node $j$. The first one is that the vehicle arrives at node $j$ before the ending of time period $T_{3}$. That is to say, $l_{i j m} \leq\left(T_{3}-T_{i k}\right) v_{3 m}$, $t_{i j k m}=l_{i j m} / v_{3 m}$. The second one is that the vehicle arrives at node $j$ after the ending of time period $T_{3}$, which crosses two time periods $T_{4}, T_{5}$. That is to say, $t_{i j k m}=\sum_{f=1}^{2} l_{i j m(3+f)} / v_{m(3+f)}$.

\subsection{Analysis of Refrigeration Cost and Cargo Damage Cost in Delivery Process}

3.3.1. Refrigeration Cost. Because fresh products are perishable, the refrigerant needs to be consumed during transportation to keep the temperature in the cabin constant [34], it will incur refrigeration cost. The refrigerant consumption during transportation is related to the temperature difference between the inside and outside of the compartment, the inside and outside surface area of the compartment, and the transportation time. The amount of refrigerant consumption during loading and unloading process is related to the heat load generated by opening and closing the door. The inside and outside temperature of the compartment is constant, and the vehicle is the same. The door only needs to be opened and closed once during loading and unloading process. Under these circumstances, the refrigeration cost during loading and unloading process is approximately positively related to the loading and unloading time. According to literature [25], the calculation of refrigeration cost is shown in the following equations:

$$
\begin{aligned}
& C^{1}=\sum_{i \in V} \sum_{j \in V} \sum_{m \in M} \sum_{k \in K}\left[\left(\rho \sqrt{s_{1} s_{2}} \Delta T_{1} \alpha+\rho \sqrt{s_{3} s_{4}} \Delta T_{2} \alpha\right) t_{i j k m} c_{5} x_{i j k m}\right] \\
& C^{2}=\sum_{i \in V} \sum_{j \in V} \sum_{0} \sum_{k \in K} t_{i k}^{s} c_{6} x_{i j k m} \\
& C_{\text {cold }}=C^{1}+C^{2}
\end{aligned}
$$

$C_{\text {cold }}$ represents the total refrigeration cost incurred during delivery, in which $C^{1}$ represents the refrigeration cost incurred during the transportation process. $C^{2}$ indicates the refrigeration cost incurred during loading and unloading process. $\Delta T_{1}$ indicates the temperature difference between inside and outside of the refrigerated compartment. $\Delta T_{2}$ indicates the temperature difference between inside and outside of the frozen compartment. $V$ indicates the thermal conductivity coefficient of the vehicle. $\theta$ indicates the heat load coefficient during loading and unloading process. $s_{1}$ indicates the outside surface area of the refrigerated vehicle body. $s_{2}$ indicates the inside surface area of the refrigerated vehicle body. $s_{3}$ indicates the inside surface area of the frozen vehicle body. $s_{4}$ indicates the outside surface area of the frozen vehicle body. $V$ indicates the volume of each compartment. $\alpha$ indicates the refrigerant consumption per unit time. $c_{5}$ indicates the unit price of the refrigerant. $c_{6}$ indicates the refrigeration cost per unit time during loading and unloading process.

3.3.2. Cargo Damage Cost. There is a fixed temperature range inside the vehicles. However, the transportation process is affected by factors such as shaking of the vehicle body and loading and unloading process; cargos will be 


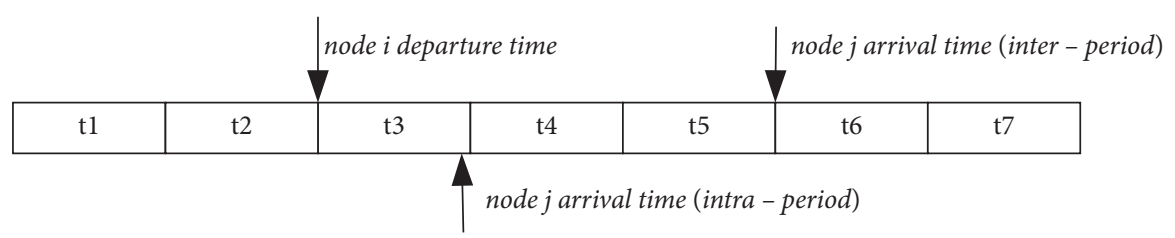

FIgURE 4: Schematic diagram of intraperiod and interperiod.

damaged during the transportation process. Therefore, we have introduced the cargo damage rate related to the compartment temperature of different kinds of fresh products. Even if the fresh products are in the appropriate temperature range, the quality of the products will deteriorate with the increase in the delivery time. Besides, when the vehicle arrives at the customer to start loading and unloading, the heat exchange inside the compartment caused by the switch of each compartment door will also affect the quality of fresh products, resulting in a certain cargo damage cost. Assuming that the temperature does not change during the delivery process, the cargo damage rate of fresh products does not change. The damage cost is mainly affected by the length of transportation time, the number of times that a door opens and closes during the loading and unloading process, the heat exchange between the inside and outside of the compartment when the door is opened and closed, and the loading and unloading time. To calculate the cargo damage rate, we have introduced the Arrhenius equation [28].

$$
\varphi=\mathrm{Ae}^{-\left(\frac{E a}{R T}\right)} .
$$

$\varphi$ represents the reaction rate constant at temperature $T, T$ is the thermodynamic temperature

$R$ is the molar gas constant, the unit is $\mathrm{kj} /(\mathrm{mol} \cdot \mathrm{k})$

$E a$ is the apparent activation energy, the unit is $\mathrm{kj} /(\mathrm{mol} \cdot \mathrm{k})$

$A$ is an antecedent factor, also known as the Arrhenius constant

It can be known from (5) that when the temperature of the compartment in which the fresh products located is different, the cargo damage rate is also different, and the cargo damage rate increases with the increase of compartment temperature. Therefore, the cargo damage cost is calculated as follows:

$$
\begin{aligned}
C^{3} & =\varphi_{1} \sum_{i \in V} \sum_{j \in V} \sum_{k \in K} \sum_{m \in M} \sum_{h \in H} t_{i j k m} c_{7} Q_{i h} x_{i j k m}+\varphi_{2} \sum_{i \in V} \sum_{j \in V} \sum_{k \in K} \sum_{m \in M} \sum_{h \in H} t_{i j k m} c_{8} Q_{i h} x_{i j k m}, \\
C^{4} & =\varphi_{1} \sum_{i \in V} \sum_{j \in V} \sum_{k \in K} \sum_{m \in M} \sum_{h \in H} t_{j k}^{s} c_{7}\left(Q_{i h}-d_{j h}\right) x_{i j k m}+\varphi_{2} \sum_{i \in V} \sum_{j \in V} \sum_{k \in K} \sum_{m \in M} \sum_{h \in H} t_{j k}^{s} c_{8}\left(Q_{i h}-d_{j h}\right) x_{i j k m}, \\
C_{\text {da mage }} & =C^{3}+C^{4},
\end{aligned}
$$

where $C_{\text {damage }}$ is the total cargo damage cost in the delivery process, $C^{3}$ is the cargo damage cost in the transportation process, $C^{4}$ is the refrigeration cost in the loading and unloading process, $c_{7}$ is the unit refrigerated cargo value, $c_{8}$ is the unit frozen cargo value, $\varphi_{1}$ is the refrigerated compartment cargo damage rate, $\varphi_{2}$ is the frozen compartment cargo damage rate, and $Q_{i h}$ is the amount of cargo in each compartment when the vehicle leaves node $i$.

\subsection{Assumption. The model assumes the following:}

(1) The vehicles are the same model and have the same performance, and the number of vehicles can meet the needs of all customers

(2) The vehicle departs from the depot and finally returns to the depot

(3) Only one vehicle will be delivered once, and each customer will only accept the service of one vehicle
(4) The locations of depot and customers and the customer demand for different cargos are known

(5) The capacity of the depot must be able to meet all customer demands

3.5. Mathematical Model. In this paper, the strategy of "preoptimization followed by real-time adjustment" is adopted to solve the dynamic multicompartment refrigerated vehicle routing problem with multigraph based on realtime traffic information.

3.5.1. Preoptimization Stage Model. As shown in Figure 5, during the preoptimization phase, the predicted travel speed functions at different times throughout the day are known. Based on the predicted travel speeds of all road sections, the transit time of different paths at a certain moment is calculated. The transit time of each path is $t_{i j k m}$; this paper selects the path with the shortest predicted arrival time during this period, so the path 1 is selected. 


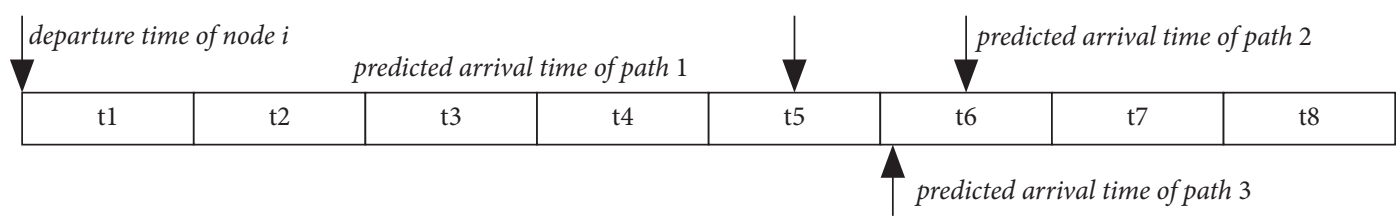

FIgURE 5: Schematic diagram of predicted arrival time.

According to the above description, the preoptimization phase model is as follows:

$$
\begin{aligned}
& \min C=c_{1} \sum_{i \in V} \sum_{j \in V_{0}} \sum_{k \in K} \sum_{m \in M} x_{i j k m} \cdot \max \left\{\left(E T_{j}-T_{j k}\right), 0\right\}+c_{2} \sum_{i \in V_{0}} \sum_{j \in V_{0}} \sum_{k \in K} \sum_{m \in M} x_{i j k m} \cdot \max \left\{\left(T_{i k}-L T_{i}\right), 0\right\} \\
& +c_{3} \sum_{i \in V} \sum_{j \in V} \sum_{k \in K} \sum_{m \in M} l_{i j m} x_{i j k m}+c_{4} \sum_{j \in V_{0}} \sum_{k \in K} x_{0 j k}+C_{c o l d}+C_{d a \text { mage }} \\
& \sum_{m \in M} \sum_{k \in K} \sum_{j \in V_{0}} x_{0 j k m} \leq|K| \\
& \sum_{m \in M} \sum_{i \in V_{0}} x_{0 i k m}=\sum_{m \in M} \sum_{j \in V_{0}} x_{j 0 k m}=1, \forall k \in K \\
& \sum_{m \in M} \sum_{k \in K} \sum_{i \in V_{0}} x_{i j k m} \leq 1, \forall j \in V \\
& \sum_{m \in M} \sum_{k \in K} \sum_{j \in V_{0}} x_{i j k m} \leq 1, \forall i \in V \\
& \sum_{i \in V} x_{i j k m}=\sum_{i \in V} x_{j i k m}, \forall j \in V_{0}, m \in M, \forall k \in K, \\
& \sum_{m \in M} \sum_{i \in V} \sum_{j \in V_{0}} \sum_{h \in H} x_{i j k m} d_{i h} \leq Q_{h} \\
& \sum_{m \in M} \sum_{i \in S} \sum_{j \in S} x_{i j k m} \leq|S|-1, \forall k \in K \\
& T_{s}+\sum_{i \in V} \sum_{j \in V} \sum_{m \in M} x_{i j k m} t_{i j k m}+\sum_{i \in V} \sum_{j \in V_{0}} \sum_{m \in M} t_{j k}^{s} x_{i j k m} \leq T_{f}, \quad \forall k \in K, \\
& \left(T_{i k}+t_{i k}^{s}+t_{i j k m}\right) x_{i j k m} \leq T_{j k}, \forall(i, j) \in V_{0}, \forall k \in K, \forall m \in M, \\
& x_{i j k m}=\{0,1\} \quad \forall i \in V, \forall j \in V, \forall k \in K, \forall m \in M .
\end{aligned}
$$

Equation (8) is the objective function, which indicates the total transportation cost, including vehicle fixed cost, transportation cost, time window penalty cost, refrigeration cost, and cargo damage cost. Equation (9) indicates that vehicles sent from the depot cannot exceed the number limit. Equation (10) indicates that the vehicle departs from the depot and returns to the depot. Equations (11) and (12) indicate that each customer is served by only one vehicle. Equation (13) ensures the balance of vehicles entering and leaving each node. It restricts the total in-degrees and out- degrees for each node. Equation (14) indicates that the service volume of each vehicle to customers shall not exceed the compartment capacity. Equation (15) is the subtour elimination constraint. Equation (16) ensures that the time when the vehicle returns to the depot does not exceed the working deadline of the depot. Equation (17) calculates the time of the vehicle arriving at node $j$ from node $i$ by sum of the arrival time of node $i$, the service time at node $i$, and the travel time from node $i$ to node $j$. Equation (18) is a decision variable attribute. 


\subsubsection{Adjust Strategies and Models in Real-Time}

Adjust Strategy in Real-Time. In the real-time adjustment stage, the order of customers in the preoptimization stage is kept unchanged. Firstly, the paths are selected according to the rules shown in Figure 6. The time of the vehicle passing through a selected path between two nodes may be different from the predicted time; when the vehicle goes to the next node, the travel time of different paths needs to be predicted again. As shown in Figure 6, the real transit time $t_{i j k m}^{z}$ and the service time of the previous node are used to calculate the real arrival time $T_{j k}^{z}$ at the node $j$. The real departure time to the next node is calculated based on the service time of the node $j$. Figure $6(\mathrm{a})$ indicates that the real arrival time is later than the predicted arrival time. Figure $6(\mathrm{~b})$ indicates that the real arrival time is earlier than the predicted arrival time. If the real arrival time is different, the real departure time and the predicted arrival time to the next node will also be different.

3.5.3. Real-Time Adjust Models. Since the calculation of cargo damage cost and refrigeration cost is related to the real transit time between two nodes, equation (6) is updated to as follows:

$$
C^{1}=\sum_{i \in V} \sum_{j \in V_{0}} \sum_{m \in M} \sum_{k \in K}\left[\left(\rho \sqrt{s_{1} s_{2}} \Delta T_{1} \alpha+\rho \sqrt{s_{3} s_{4}} \Delta T_{2} \alpha\right) t_{i j k m}^{z} c_{5} x_{i j k m}\right] .
$$

Equation (9) is updated to as follows:

$$
C^{3}=\varphi_{1} \sum_{i \in V} \sum_{j \in V_{0}} \sum_{k \in K} \sum_{m \in M} \sum_{h \in H} t_{i j k m}^{z} c_{7} Q_{i h} x_{i j k m}+\varphi_{2} \sum_{i \in V} \sum_{j \in V_{0}} \sum_{k \in K} \sum_{m \in M} \sum_{h \in H} t_{i j k m}^{z} c_{8} Q_{i h} x_{i j k m} .
$$

Objective function:

$$
\begin{aligned}
\min Z= & c_{1} \sum_{i \in V} \sum_{j \in V_{0}} \sum_{k \in K} \sum_{m \in M} x_{i j k m} \cdot \max \left\{\left(E T_{j}-T_{j k}\right), 0\right\}+c_{2} \sum_{i \in V_{0}} \sum_{j \in V_{0}} \sum_{k \in K} \sum_{m \in M} x_{i j k m} \cdot \max \left\{\left(T_{i k}-L T\right)_{i}, 0\right\} \\
& +c_{3} \sum_{i \in V} \sum_{j \in V} \sum_{k \in K} \sum_{m \in M} l_{i j m} x_{i j k m}+C_{c o l d}+C_{\text {da mage }} .
\end{aligned}
$$

Equations (9)-(16) and (18),

$\left(T_{i k}^{z}+t_{i k}^{s}+t_{i j k m}^{z}\right) x_{i j k m} \leq T_{j k}^{z}, \forall(i, j) \in V_{0}, \forall k \in K, \forall m \in M$.

Equation (21) is the objective function of the real-time adjustment stage, including transportation cost, time window penalty cost, refrigeration cost, and cargo damage cost. Equations (9)-(16) and (18) are the same as the preoptimization stage. Equation 22 calculates the arrival time of node $j$ from node $i$ by the sum of the arrival time of node $i$, the service time at node $i$, and the travel time from node $i$ to node $j$.

\section{Solution Approach}

The dynamic multicompartment refrigerated vehicle routing problem with multigraph based on real-time traffic information studied in this paper considers such factors as multicompartment refrigerated vehicle, multigraph, time window, real-time traffic information, and so on. As an extension of classical VRP, it also belongs to NP-hard problem. It cannot obtain an optimal solution within a reasonable calculation time, so it is essential to solve it with a heuristic algorithm. The proof of NP-hard problem is as follows.

Theorem 1. The dynamic multicompartment refrigerated vehicles routing problem with multigraph based on real-time traffic information is an NP-hard problem.

Proof. This model is an extension of Garaix et al. [11] to multicompartment refrigerated vehicle, time window, and real-time traffic information problem. As it is presented by Garaix et al. [11], the fixed sequence arc selection problem (FSASP) is NP-hard. So, the model proposed in this paper is NP-hard as well. Furthermore, there are several papers that the multicompartment vehicle routing problems are NPhard (Kaabachi et al. [22], Ostermeier and Hübner [24], and Coelho and Leandro [29]).

Genetic algorithm (GA) is a heuristic algorithm based on genetic theory and natural selection, which has been widely used in vehicle routing problems [38]. It combines the survival rules of the fittest and the random exchange mechanism of genetic information in the population in the 


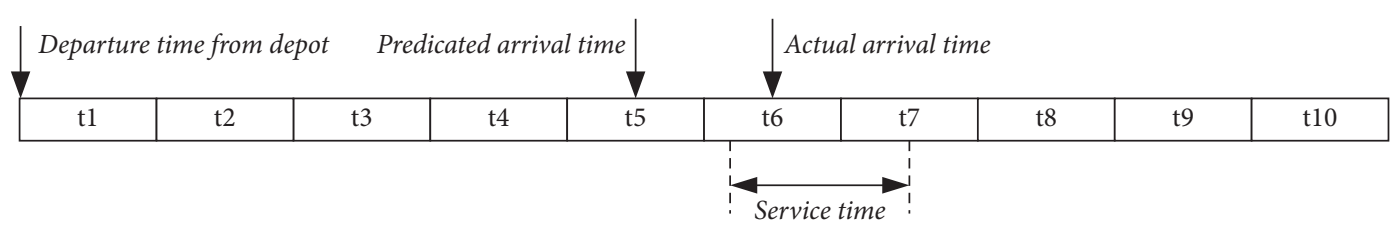

(a)

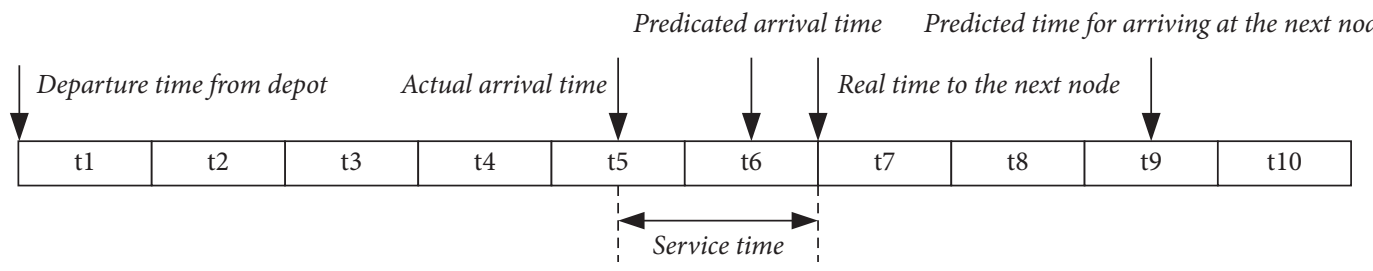

(b)

FIgURE 6: Schematic diagram of real-time calculation. (a) Later than the predicted arrival time. (b) Earlier than the predicted arrival time.

process of biological evolution. It has good robustness and global search performance. However, the algorithm is easy to converge prematurely, and the initial population of the traditional genetic algorithm is mostly generated randomly. Most seemingly random species in real biological systems follow certain rules-genetic theory. Aiming at this characteristic, this paper initializes the population through the chaos phenomenon. The variable neighborhood search algorithm uses multiple different neighborhood structures for systematic search and has a strong local search capability. Therefore, this paper combines a genetic algorithm and variable neighborhood search algorithm and designs an improved hybrid chaotic genetic algorithm with variable neighborhood search algorithm based on the idea of preoptimization followed by real-time adjustment. In the preoptimization stage, this algorithm is used to obtain the optimal delivery route and path selection scheme. In the real-time adjustment stage, the optimal path is reoptimized and is selected at the customer according to the real-time traffic information to minimize the loss of fresh products. The algorithm flow is shown in Figure 7.

4.1. Encoding and Initial Population Generation. The algorithm in this paper adopts the form of natural number coding. Since the type of customer demand is uncertain, when generating the initial population, the initial value of logistic mapping is generated randomly according to the population size. The chaotic system of each group (each group consists of $n$ values) is calculated by equation (23), and the initial population is generated according to the position of $n$ values in the chaotic system of each group.

$$
x_{n+1}=r x_{n}\left(1-x_{n}\right), n=1,2, \cdots, r \in(3.57,4], x_{i} \in[0,1] .
$$

The purpose of studying chaos is to reveal the laws generally followed by some complex problems that may be hidden behind seemingly random, chaotic, and disordered phenomena. In a given chaotic equation $y=f(x), x \in[a, b]$, if there is an initial value $x_{0} \in[a, b]$, making $f(0)=x_{0}$, then $x_{0}$ is said to be the fixed point of $y$. In the logistic mapping, $x_{0}=0.25,0.5,0.75$ are fixed points, and Figure 8 shows the nature of the fixed point. As can be seen from Figure 8 , when the initial value $x_{0}$ is a fixed point, the system loses ergodicity. If the initial value of the logistic mapping is $x_{0} \in[0,1]$ and $x_{0} \neq 0.25,0.5,0.75$, the pseudorandom number $x_{i} \in[0,1 / 4 r]$ is generated by multi-iteration. When $r=4$, any $x_{i} \in[0,1]$ is always established, and when $r=4$, the system is in a completely chaotic state, it has all the characteristics of a chaotic system, so the value of chaotic coefficient $r$ in this paper is 4 .

After the initial population is generated according to the chaos principle, the depot is inserted before the first customer, and then the depot is inserted into the customer arrangement by using the nearest neighbor insertion method. When the sum of the demand for exactly one kind of cargos at a customer exceeds the compartment capacity, the insertion operation is performed. The insertion position of each " 0 " is recorded. It will arrange vehicles according to the number and location of "0," as shown in Figure 9. From Figure 9, the route served by each vehicle can be confirmed only by recording the location of each depot, i.e., the service sequence of the first vehicle is 3-7-4, and the service sequence of the second vehicle is 2-1-5. By using this method, the feasibility of generating the initial solution can be ensured, and the problems of unfixed coding length and difficulty in repairing the infeasible solution can be avoided.

4.2. Fitness. The fitness function of chromosomes can be constructed according to objective function. The fitness function of a chromosome $s$ can be expressed as follows:

$$
f_{S}=\frac{1}{z}
$$

where $z$ is the objective function value of the chromosome $s$.

4.3. Selection. The selection operation adopts the strategy of combining elite selection with roulette. The specific steps are as follows: firstly, the individuals are ranked in descending order of fitness value. Then, some elite individuals are 


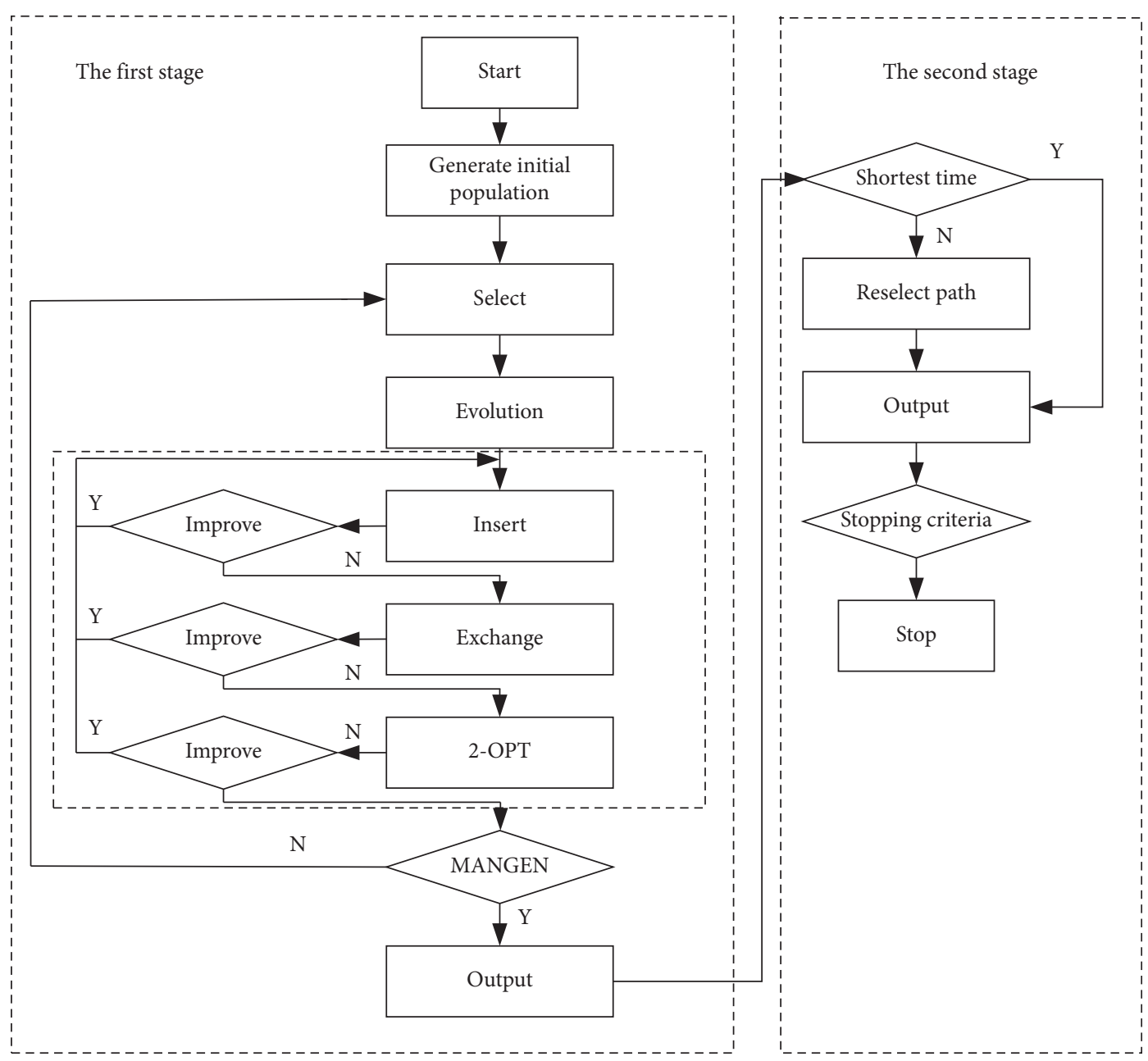

Figure 7: HCGAVNS flowchart.

reserved, and the remaining individuals are reserved by roulette. The probability of everyone being selected is proportional to the fitness function value, i.e., the higher the fitness function value, the higher the probability of the individual being selected. On the contrary, the lower the probability of being selected. When each generation of disturbance is over, the elite individuals are compared with the optimal offspring. If the elite individuals are superior to the offspring, the offspring are directly replaced; otherwise, the elite individuals are updated.

4.4. Evolution. The evolutionary operation in this paper adopts a sequential crossover operator. As shown in Figure 10, when parent $\mathrm{A}$ is crossed sequentially, parent $\mathrm{B}$ is randomly selected from the population. Firstly, points $i_{11}$, $i_{12}, i_{21}$, and $i_{22}$ are randomly generated; the part between $i_{11}$ and $i_{12}$ of the parent $\mathrm{A}$ is taken as the first segment of the subgeneration. Al and the subsequent points of the subgeneration $\mathrm{A} 1$ are related to parent $\mathrm{B}$, i.e., the customers between $i_{11}$ and $i_{12}$ in the parent $\mathrm{B}$ are eliminated first. In the elimination process, the position sequence of the customers in the parent $\mathrm{B}$ is not changed, and then the eliminated customers are arranged as the second segment of the subgeneration $\mathrm{A} 1$ to form the subgeneration $\mathrm{A} 1$ and the subgeneration $\mathrm{B} 1$.

\subsection{Local Search Strategy}

4.5.1. Neighborhood Structure. Firstly, neighborhood structure sets $N_{k}=\left\{N_{1}, N_{2}, \ldots N_{l}\right\}$ are constructed; the individual $x$ in the population starts to be disturbed from the first neighborhood structure $N_{1}$. If no improved solution is found within the preset neighborhood search times $S_{n}$, the next neighborhood structure is executed. Otherwise, if an improved solution $x^{\prime}$ is obtained in a certain neighborhood structure, then $x=x^{\prime}$ is made, and the iteration is restarted by returning to the first neighborhood structure. Until the iteration is cycled to the last neighborhood structure, and when the improved solution is not found, the search is terminated. When the number of variable neighborhood search cycles reaches the preset $\operatorname{MaxS}_{n}$, the search is terminated, and the algorithm enters the next stage. In this paper, three neighborhood structures are used to enhance the local search capability of the algorithm.

(1) Insert: randomly select customers $i$ and $j$ from the chromosome and insert $i$ after $j$. As shown in 

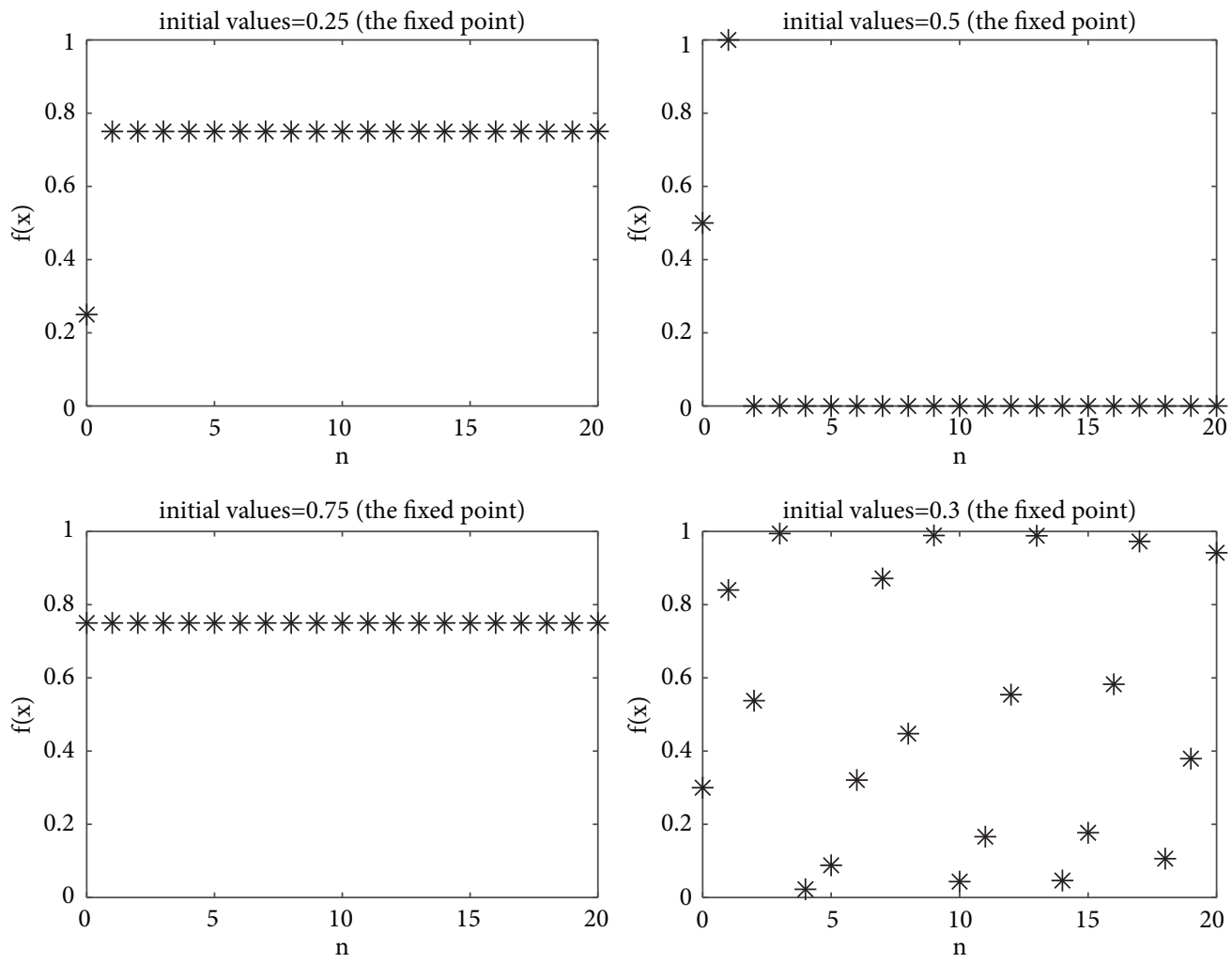

FIGURE 8: Fixed points of logistic mapping.

\begin{tabular}{c}
$\qquad$\begin{tabular}{|c|c|c|c|c|c|c|c|c|c|}
\hline \multicolumn{1}{|c|}{3} & 7 & 4 & 2 & 1 & 5 & 6 & 8 & \\
\hline 0 & 3 & 7 & 4 & 0 & 2 & 1 & 5 & 0 & 8 \\
\hline
\end{tabular} \\
\hline
\end{tabular}

FIgURE 9: Schematic diagram of the encoding mode.
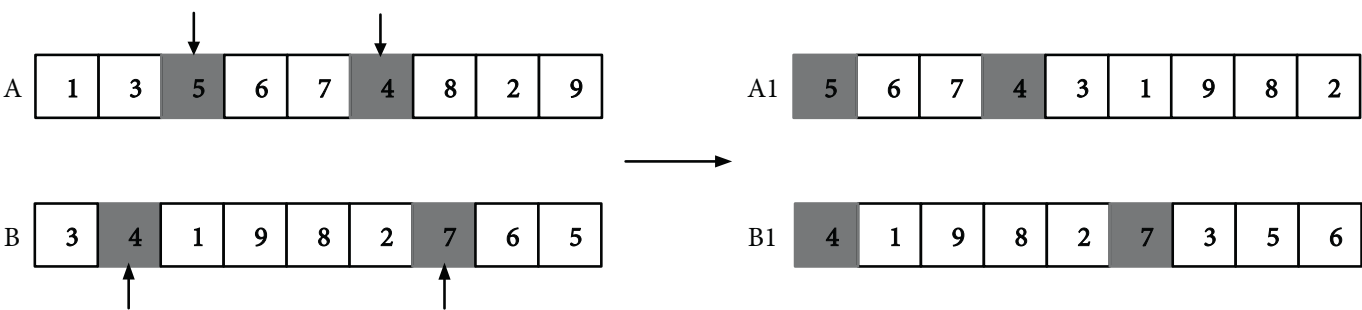

$\mathrm{B} 1$

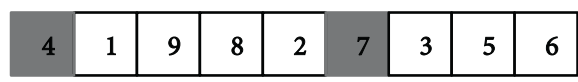

Figure 10: Schematic diagram of a sequential crossover operator.

Figure 11(a), customer 3 and customer 6 are selected randomly; customer 3 is inserted after customer 6 .

(2) Exchange: randomly select customer $i$ and $j$, then exchange their positions. As shown in Figure 11(b), customer 3 and customer 6 are selected randomly; customer 3 and customer 6 exchanged locations.

(3) 2-OPT: randomly select customers $i$ and $j$ and exchange the order of other customers among customers $i$ and $j$. As shown in Figure 11(c), the position of customer 3 is kept unchanged, and customers $4,5,7$, and 6 are in reverse order.

4.5.2. Adaptive Mechanism. In this paper, an adaptive neighborhood search number strategy is proposed to enhance the breadth and depth of the algorithm search so that the variable neighborhood search algorithm can jump out of 


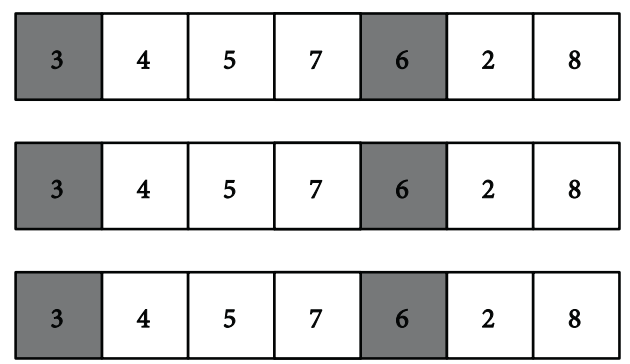

FIgURE 11: Neighborhood structures.

\begin{tabular}{|c|c|c|c|c|c|c|c|}
\hline $\begin{array}{l}\text { (a) Ins } \\
\text { Insert }\end{array}$ & 4 & 5 & 7 & 6 & 3 & 2 & 8 \\
\hline & & & & & & & \\
\hline xchange & 6 & 4 & 5 & 7 & 3 & 2 & 8 \\
\hline (c) $2-\mathrm{O}$ & & & & & & & \\
\hline $\mathrm{OPT}$ & 3 & 6 & 7 & 5 & 4 & 2 & 8 \\
\hline
\end{tabular}

(a) Insert. (b) Exchange. (c) 2-OPT. the local optimization. Neighborhood search times have a great influence on the searchability of the algorithm, which directly leads to the performance of the algorithm. In the iterative process of the algorithm, the disturbance intensity required by the population is different. At the beginning of the iteration, the number of neighborhood searches should be small to make the population converge quickly. However, with the continuous iteration of the population, the number of neighborhood searches is increased to enhance the search capability of the algorithm. The adaptive neighborhood search number strategy in this paper is as follows:

(1) Setting the initial neighborhood search number $S_{n}=$ 1 and the number of times that the optimal solution is continuously unchanged con num.

(2) If the optimal solution of the population after this iteration is not improved, let con num $=$ con num $+1, S_{n}=S_{n}+1$. If the perturbed solution is improved, let con $n u m=0$, $S_{n}=1$.

(3) When the number of times con num that the optimal solution has not changed continuously until the preset value stop_num, the algorithm terminates and outputs the optimal solution.

4.6. Calculation of Double-Loop Objective Function. When the real departure time and the predicted departure time of the same road section are different, the speed of the vehicle will be different, and the travel time of the same road section will be different in the first and second phases. Therefore, in the second phase of the solution, this paper designs a double-loop iteration to realize the route selection with the shortest travel time and the calculation of the cost. The specific process is shown in Figure 12. The inner cycle calculates the travel time by calling in the predicted traffic data and the distance matrix corresponding to different roads, and the outer cycle starts by comparing the recorded travel times of different paths and calls the traffic data of the real-time data platform at the same time and records the real-time of the selected path and the time period when it reaches the customer, thus calculating various costs, and takes the time when it reaches the customer as the new departure time for the next loop.

4.7. Time Complexity Analysis of the Algorithm. This section analyzes the time complexity of the algorithm. Assume that the customer scale is $n$, the population size is pop_size, and the maximum number of iterations is $\max _{-}$gen. The time complexity of the algorithm is calculated as follows: (1) generate initial population. $O\left(T_{1}\right)=$ pop_size; (2) fitness function calculation. $O\left(T_{2}\right)=$ max $_{-}$gen; (3) selecting operation. $O\left(T_{3}\right)=\max _{-} g e n ;$ (4) evolution operation. $O\left(T_{4}\right)=n \cdot$ pop_size $\cdot$ max $_{-} g e n ;(5)$ variable neighborhood search (including three neighborhood structures): $O\left(T_{5}\right)=3 n \cdot$ pop_size $\cdot$ stop num; (6) calculation of double-loop objective function. $O\left(T_{6}\right)=n \cdot$ pop_size.stop num. To sum up, the time complexity of the algorithm can be calculated as $O(T)=$ pop_size $\cdot\left(1+n \cdot \max { }_{-} g e n+3 n\right.$ stop_num $)+2 \max _{-} g e n$.

\section{Numerical Experiments}

5.1. Example Description. There is no general example set at present due to the excessive related constraints and multigraph characteristics of cold chain delivery vehicles under the dynamic route delivery mode. The real-time information of the road network in this paper uses the data published by the University of Minnesota. It randomly selects $n$ groups historical data for prediction and generates $n$ kinds of different speed information. This paper adopts A-n45-k6 in the international standard instance library, which has one depot and forty-four customers. The specific information is shown in Table 1 . The customer demand is multiplied by 0.1 based on the standard instance and divided into two types according to the ratio of $2: 1$. The unit of customer demand is ton, and the customer demand in the instance is the quantity on a given day. Assuming that there are three paths between two nodes, the distance matrix is expanded from one to three, in which the position coordinates are unchanged, and the distance of different paths between nodes is randomly multiplied by the distance coefficient [17]. The coefficient value is changing from 0.8 to 1.2. There are two types of cargo to be delivered, and the temperature required for refrigerated cargos is $0^{\circ} \mathrm{C}$; the frozen cargos temperature is $-10^{\circ} \mathrm{C}$. The external temperature is $20^{\circ} \mathrm{C}$. We select Dongfeng refrigerated commercial vehicle (DFH5160XLCEX2B) as the transport vehicle. It has a payload capacity of $8 \mathrm{t}$, a compartment size of $8 \times 2.44 \times 2.4 \mathrm{~m}$, and the fuel consumption of $25.9 \mathrm{~L}$ per hundred kilometers. A compartment is divided into two compartments in the form of an intermediate compartment partition according to a volume ratio of $2: 1$. The working time window of the depot is $6: 00-19$ : 00 . The remaining parameters are shown in Table 2 . 


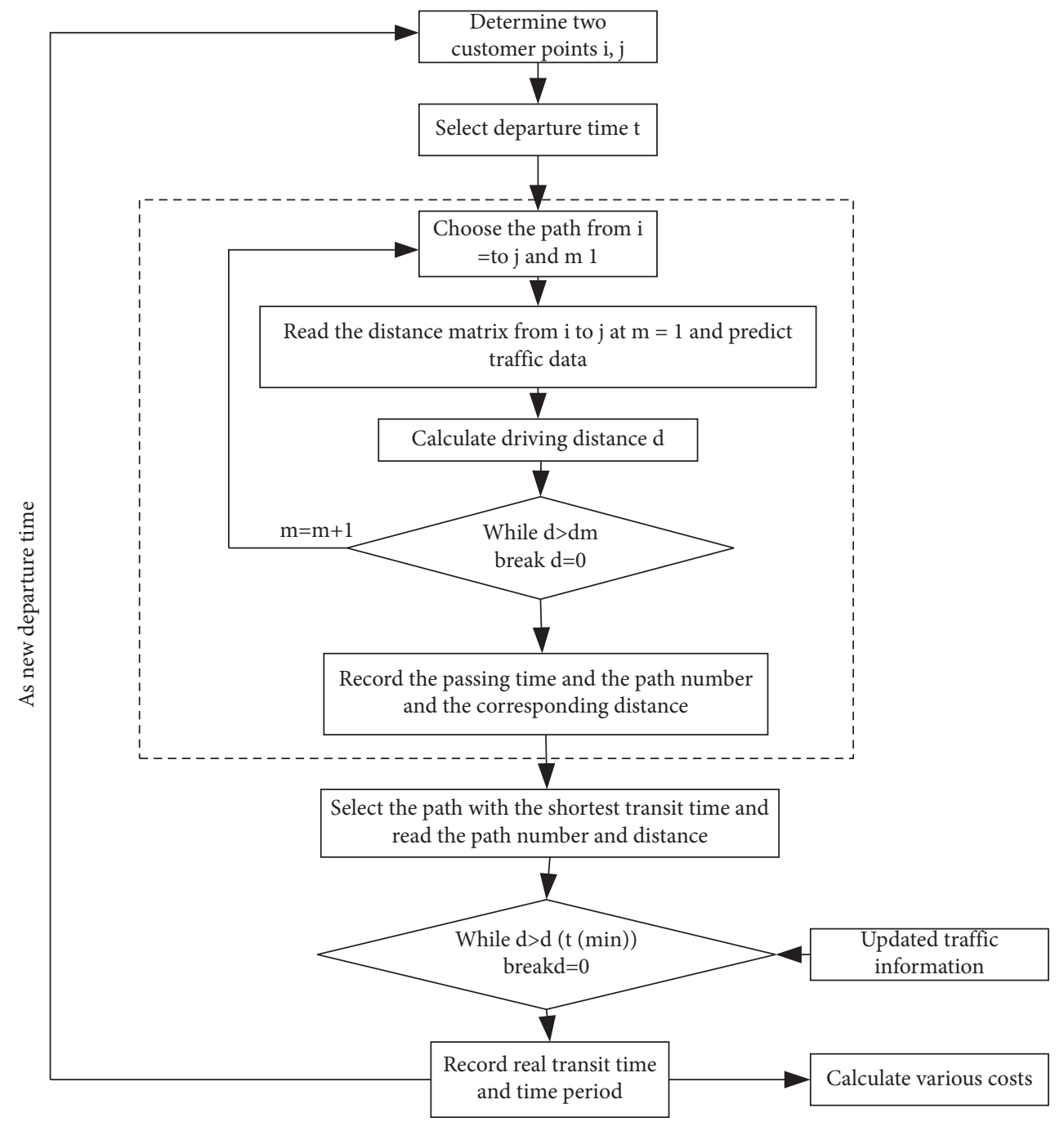

FIgURE 12: Double-loop objective function solution.

TABle 1: Data in the instance.

\begin{tabular}{lcccccccccc}
\hline No. & 1 & 2 & 3 & 4 & 5 & 6 & 7 & 8 & 9 \\
\hline$X$ & 31 & 11 & 52 & 81 & 97 & 71 & 6 & 48 \\
$Y$ & 73 & 67 & 96 & 29 & 62 & 5 & 56 & 50 \\
Time window & {$[6,19]$} & {$[6.5,10]$} & {$[7.5,16.5]$} & {$[8.5,15.5]$} & {$[7.5,16.5]$} & {$[8.5,13.5]$} & {$[7.5,16.5]$} & {$[8.5,13.5]$} & {$[7.5,16.5]$} \\
No. & 10 & 11 & 12 & 13 & 14 & 15 & 16 & 17 & 18 \\
$X$ & 49 & 85 & 11 & 74 & 56 & 13 & 66 & 96 & 36 \\
$Y$ & 68 & 29 & 16 & 98 & 37 & 81 & 80 & 55 & 17 \\
Time window & {$[6.5,9.5]$} & {$[8.5,13.5]$} & {$[6.5,10]$} & {$[7.5,16.5]$} & {$[8.5,13.5]$} & {$[7.5,16.5]$} & {$[8.5,13.5]$} & {$[7.5,16.5]$} & {$[7.5,15.5]$} \\
No. & 19 & 20 & 21 & 22 & 23 & 24 & 25 & 26 \\
$X$ & 32 & 6 & 64 & 87 & 75 & 40 & 1 & 60 \\
$Y$ & 23 & 13 & 30 & 5 & 61 & 72 & 44 & 95 \\
Time window & {$[6.5,10.5]$} & {$[6.5,9.5]$} & {$[7.5,16.5]$} & {$[7.5,15.5]$} & {$[6.5,9.5]$} & {$[7.5,16.5]$} & {$[6.5,12]$} & {$[7.5,12]$} & {$[7.5,16.5]$} \\
No. & 28 & 29 & 30 & 31 & 32 & 33 & 34 & 35 \\
$X$ & 15 & 46 & 28 & 3 & 1 & 53 & 98 & 6 \\
$Y$ & 33 & 53 & 43 & 9 & 100 & 46 & 8 & 27 \\
Time window & {$[7.5,16.5]$} & {$[6.5,16.5]$} & {$[8.5,13.5]$} & {$[6.5,12]$} & {$[8.5,13.5]$} & {$[7.5,16.5]$} & {$[6.5,12]$} & {$[7.5,15.5]$} & {$[6.5,10.5]$} \\
No. & 37 & 38 & 39 & 40 & 41 & 42 & 43 & 44 \\
$X$ & 96 & 2 & 32 & 95 & 9 & 96 & 90 & 45 \\
$Y$ & 88 & 35 & 94 & 94 & 11 & 16 & 68 & 33 \\
Time window & {$[6.5,9.5]$} & {$[7.5,12]$} & {$[7.5,16.5]$} & {$[8.5,14]$} & {$[7.5,15.5]$} & {$[7.5,16.5]$} & {$[6.5,12]$} & {$[7.5,16.5]$} & {$[7.5,16.5]$} \\
\hline
\end{tabular}


5.2. Algorithm Test. After repeated tests, the parameters of this algorithm are set as follows: population size pop_size $=30 \sim 150, \quad$ maximum iteration number max_gen $=800$, initial variable neighborhood search number $S_{n}=1$, maximum neighborhood cycle number $\operatorname{MaxS}_{n}=1000$, and preset value stop num $=20 \sim 50$. The value of the parameter is related to the customer scale $n$, when $n \leq 50$, pop_size $=30$, and stop num $=20$, when $50<n \leq 100$, pop_size $=100$, and stop num $=30$, and when $n>100$, pop_size $=150$, and stop num $=50$.

To verify the performance of HCGAVNS, we calculate the $\mathrm{C} 1 \mathrm{~A}$ data provided by reference [40] and compare the results with ant colony optimization (ACO) [40], adaptive genetic algorithm (AGA), improved adaptive genetic algorithm (IAGA), artificial bee colony (ABC), and particle swarm optimization (PSO) [18]. The results are shown in Table 3. Figure 13 is the optimal circuit diagram for this instance, and Figure 14 is the optimal circuit diagram of reference [40].

As can be seen from Table 3, the results of HCGAVNS are better than those of other algorithms, and the improvement ranges of other five algorithms are $1.6 \%, 3.1 \%$, $2.3 \%, 0.7 \%$, and $0.5 \%$ respectively. Moreover, as can be seen from Figures 13 and 14, the number of customers served by each vehicle in reference [40] is quite different, with each vehicle serving at least 2 customers and up to 12 customers. The maximum number of customers served by per vehicle in this paper is 11 and at least 4, which is more in line with the actual status of logistics services.

To further verify the effectiveness of the algorithm proposed in this paper in solving different scales instances, PSO, ABC, a hybrid genetic algorithm with variable neighborhood search (HGAVNS), and HCGAVNS proposed in this paper are applied to 14 instances in the VRP international standard instance library in the same adaptation way as the C1A instance in reference [40]. The optimal results and mean values are shown in Table 4, where the bolded items are relatively better in comparison, and the deviation of the optimal solution is shown in Table 5.

From Table 4, we can see that among the four algorithms, 16 data solved by HCGAVNS are better than other algorithms, and most of the optimal results are improved. From Table 5, we can see that the maximum deviation of the optimal solution of this algorithm is $4.1 \%$ and the minimum deviation is $0.9 \%$, and the average deviation of the optimal solution is $2.1 \%$, which is more stable than PSO and ABC. Therefore, we can see that the algorithm designed in this paper has a good optimization ability for different scale instances and the effectiveness and applicability of this algorithm have been verified again.

5.3. Example Comparison. The instance in this paper is modified to reduce the number of paths of two nodes to 1 in the case of multicompartment, and the distance matrix of single path is directly calculated by the standard instance. Under the same assumptions and system optimization environment, the HCGAVNS algorithm designed in this paper is used to solve the multigraph delivery and the single-graph
TABLE 2: Parameter values.

\begin{tabular}{lccccc}
\hline Symbol & Value & Reference & Symbol & Value & Reference \\
\hline$c_{1}$ & 50 & Reference [34] & $c_{2}$ & 100 & Reference [34] \\
$c_{4}$ & 150 & Reference [34] & $c_{5}$ & 26 & Approximate \\
$c_{7}$ & 6000 & Approximate & $c_{8}$ & 18000 & Approximate \\
$\rho$ & 0.024 & Approximate & $c_{3}$ & 1.95 & Approximate \\
$\alpha$ & 0.14 & Reference [39] & $c_{6}$ & 12 & Reference [34] \\
\hline
\end{tabular}

TABLE 3: Results of various algorithms.

\begin{tabular}{lcccccc}
\hline No. & ABC & AGA & IAGA & $\begin{array}{c}\text { ACO } \\
{[40]}\end{array}$ & PSO [18] & HCGAVNS \\
\hline $\begin{array}{l}\text { Best } \\
\text { Dev } \\
\%\end{array}$ & 566.18 & 574.85 & 570.23 & 560.74 & 559.84 & 556.81 \\
\hline
\end{tabular}

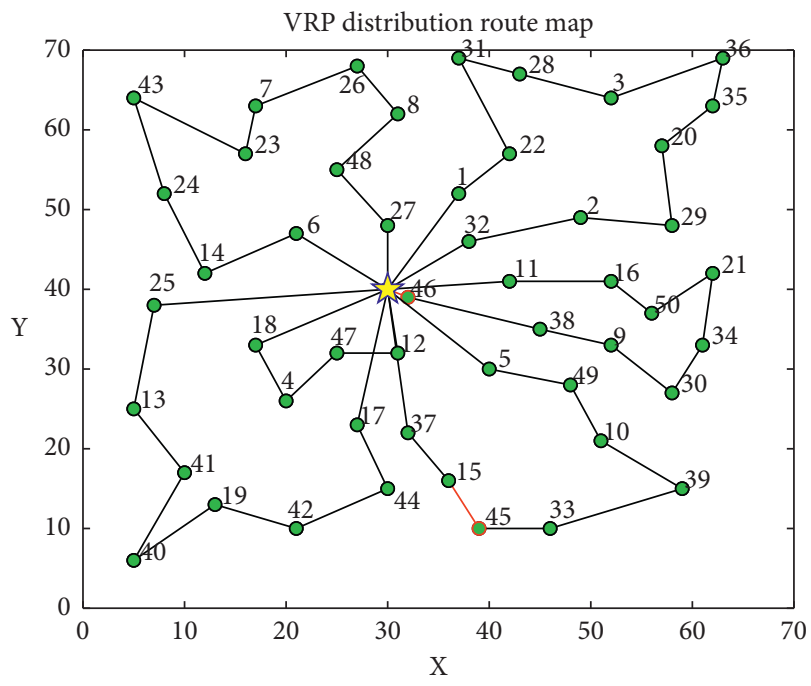

Figure 13: The optimal circuit diagram in this paper.

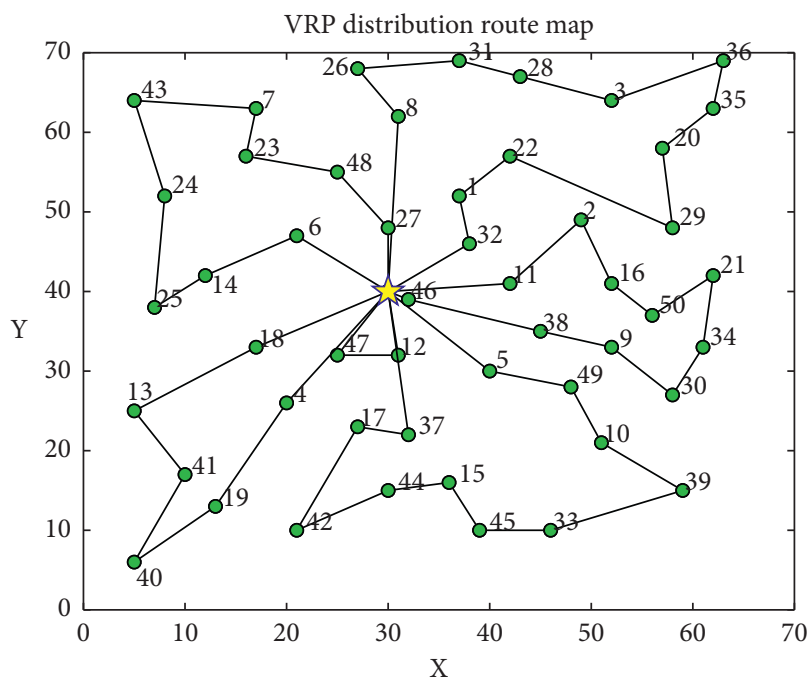

FIgURE 14: Reference [40] optimal circuit diagram. 
TABLE 4: Comparison of optimal results and average values of different algorithms.



TABLE 5: The optimal solution deviation values of each algorithm.

\begin{tabular}{lcccc}
\hline & PSO (\%) & ABC (\%) & HGAVNS (\%) & HCGAVNS (\%) \\
\hline VRPNC1 & 2.9 & 1.1 & 1.7 & 2.6 \\
VRPNC2 & 1.0 & 1.0 & 1.1 & 1.6 \\
VRPNC3 & 2.3 & 0.8 & 1.6 & 1.3 \\
VRPNC4 & 2.3 & 1.1 & 1.1 & 1.6 \\
VRPNC5 & 1.8 & 2.2 & 0.9 & 1.6 \\
VRPNC6 & 1.7 & 1.5 & 2.2 & 1.8 \\
VRPNC7 & 1.0 & 1.2 & 1.1 & 1.8 \\
VRPNC8 & 2.3 & 1.5 & 1.1 & 1.4 \\
VRPNC9 & 2.3 & 1.4 & 0.8 & 0.9 \\
VRPNC10 & 1.8 & 1.1 & 1.0 & 2.7 \\
VRPNC11 & 1.9 & 3.2 & 1.9 & 2.5 \\
VRPNC12 & 5.4 & 3.8 & 4.3 & 2.8 \\
VRPNC13 & 4.4 & 4.1 & 2.3 & 4.1 \\
VRPNC14 & 4.1 & 4.8 & 4.5 & 3.2 \\
Average & 2.5 & 2.1 & 1.8 & 2.1 \\
\hline
\end{tabular}

delivery problem ten times, and the results of ten times are listed in Table 6.

As can be seen from Table 6, in the case of multigraph, the optimal solution of the scheme is 7695.50 , the cargo damage cost is 1136.10 , the worst solution is 8460.80 , the cargo damage cost is 1087.30 , and the average value is 8141.48. Compared with this, the deviation of the optimal solution is $5.4 \%$, the deviation of the worst solution is $3.9 \%$, and the solution results are relatively stable. In the case of single graph, the optimal solution of the scheme is 8214.30, and the cargo damage cost is 1097.80 . The worst solution is 9264.00, and the cargo damage cost is 1397.70. The average value is 8697.79. Compared with this, the deviation of the optimal solution is $5.4 \%$, the deviation of the worst solution is $6.5 \%$, and the convergence result is also relatively stable. Through comparison, it can be seen that the total service cost of the multigraph strategy is reduced by $6.3 \%$, and the cargo damage cost is reduced by $4.2 \%$. It is not difficult to find that the multigraph strategy can reduce the delivery cost, improve the delivery efficiency of fresh products logistics e-commerce providers, and reduce the resource limitation of refrigerated vehicles. The delivery routes corresponding to
TABLE 6: Multigraph and single-graph problem results.

\begin{tabular}{lcccc}
\hline & \multicolumn{2}{c}{ Multigraph } & \multicolumn{2}{c}{ Single graph } \\
No. & $\begin{array}{c}\text { Total } \\
\text { cost }\end{array}$ & $\begin{array}{c}\text { Cargo damage } \\
\text { cost }\end{array}$ & $\begin{array}{c}\text { Total } \\
\text { cost }\end{array}$ & $\begin{array}{c}\text { Cargo damage } \\
\text { cost }\end{array}$ \\
\hline 1 & 7695.50 & 1136.10 & 9264.00 & 1397.70 \\
2 & 7949.30 & 1070.20 & 8856.30 & 1268.00 \\
3 & 8273.60 & 1202.90 & 9021.30 & 1250.10 \\
4 & 7969.20 & 1164.90 & 8706.30 & 1157.40 \\
5 & 7970.50 & 1270.70 & 8214.30 & 1097.80 \\
6 & 8455.70 & 1193.80 & 8336.60 & 1123.60 \\
7 & 8371.40 & 1217.20 & 8640.20 & 1179.50 \\
8 & 8460.80 & 1087.30 & 8413.70 & 1332.50 \\
9 & 8136.00 & 1226.30 & 8750.40 & 1062.40 \\
10 & 8132.80 & 1026.90 & 8774.80 & 1237.30 \\
Average & 8141.48 & 1159.63 & 8697.79 & 1210.63 \\
Best & 7695.50 & 1136.10 & 8214.30 & 1097.80 \\
Worst & 8460.80 & 1087.30 & 9264.00 & 1397.70 \\
\hline
\end{tabular}

the optimal solution in the preoptimization stage are shown in Table 7.

Due to the local adjustment strategy adopted in this paper, only the secondary selection of paths between two nodes is reconducted based on preoptimization, so the order of nodes in the real-time adjustment stage is the same as in the preoptimization stage. The route and information of the minimum total cost of the dynamic multicompartment refrigerated vehicle routing problem with multigraph based on updated traffic information are shown in Table 8.

From Tables 7 and 8 , the route selection based on the real-time traffic information can reduce the cost of cargo damage and reduce the delivery time to continuously meet the strict requirements of customers on fresh products delivery. To sum up, the algorithm designed in this paper can stably solve the dynamic multicompartment refrigerated vehicle routing problem with a multigraph based on realtime traffic information.

To verify the effectiveness of the algorithm presented in this paper in terms of solving efficiency, the solution results 
TABLE 7: The delivery routes corresponding to the optimal solution in the preoptimization stage.

\begin{tabular}{|c|c|c|c|c|c|}
\hline & Route & Selected path & Delivery time $(h)$ & Total cost & Cargo damage cost \\
\hline Vehicle1 & $0-39-15-22-7-8-41-0$ & $2,3,1,1,1,1,1$ & 5.70 & 1382.60 & 196.19 \\
\hline Vehicle2 & $0-31-38-14-35-0$ & $2,1,1,1,1$ & 3.61 & 716.73 & 130.83 \\
\hline Vehicle3 & $0-1-37-19-40-11-0$ & $2,1,1,1,1,1$ & 3.24 & 660.25 & 89.05 \\
\hline Vehicle4 & $0-29-44-6-24-0$ & $3,1,3,3,1$ & 2.79 & 650.47 & 113.91 \\
\hline Vehicle5 & $0-12-36-42-4-16-0$ & $3,1,1,1,1,1$ & 3.32 & 790.12 & 135.07 \\
\hline Vehicle6 & $0-25-2-20-33-21-17-0$ & $3,1,3,1,1,1,1$ & 5.80 & 1180.20 & 215.74 \\
\hline Vehicle7 & $0-10-5-3-13-32-9-26-0$ & $3,1,1,1,1,1,1,1$ & 5.41 & 1093.30 & 146.16 \\
\hline Vehicle8 & $0-43-18-27-34-30-0$ & $2,1,1,1,1,1$ & 3.06 & 863.93 & 85.58 \\
\hline Vehicle9 & $0-23-28-0$ & $3,1,1$ & 1.36 & 357.94 & 23.61 \\
\hline Total & - & - & - & 7695.5 & 1136.1 \\
\hline
\end{tabular}

TABle 8: Optimization results of delivery routes.

\begin{tabular}{lcccc}
\hline & Route & Selected path & $\begin{array}{c}\text { Delivery } \\
\text { time }(h)\end{array}$ & Cargo damage cost \\
\hline Vehicle1 & $0-39-15-22-7-8-41-0$ & $1,3,2,2,2,2,1$ & 5.7 & 197.24 \\
Vehicle2 & $0-31-38-14-35-0$ & $2,1,2,2,1$ & 3.52 & 128.12 \\
Vehicle3 & $0-1-37-19-40-11-0$ & $2,1,1,2,2,1$ & 3.07 & 85.58 \\
Vehicle4 & $0-29-44-6-24-0$ & $3,1,2,2,1$ & 2.75 & 112.27 \\
Vehicle5 & $0-12-36-42-4-16-0$ & $3,1,2,2,1,1$ & 3.32 & 135.24 \\
Vehicle6 & $0-25-2-20-33-21-17-0$ & $3,1,2,2,2,2,1$ & 5.85 & 216.84 \\
Vehicle7 & $0-10-5-3-13-32-9-26-0$ & $3,1,2,2,2,2,2,1$ & 5.49 & 146.09 \\
Vehicle8 & $0-43-18-27-34-30-0$ & $2,1,1,2,2,1$ & 3 & 83.46 \\
Vehicle9 & $0-23-28-0$ & $3,1,1$ & 1.35 & 23.79 \\
Total & - & - & - & 1128.62 \\
\hline
\end{tabular}

TABle 9: Comparison of the three algorithms.

\begin{tabular}{|c|c|c|c|c|c|c|c|c|c|}
\hline \multirow{2}{*}{ No. } & \multicolumn{3}{|c|}{ AGA } & \multicolumn{3}{|c|}{ IAGA } & \multicolumn{3}{|c|}{ HCGAVNS } \\
\hline & Total cost & Cargo damage cost & $\mathrm{CPU}$ & Total cost & Cargo damage cost & $\mathrm{CPU}$ & Total cost & Cargo damage cost & CPU \\
\hline 1 & 8914.2 & 1167.7 & 104.81 & 8445.6 & 1174.5 & 104.20 & 7695.50 & 1136.10 & 78.73 \\
\hline 2 & 8249.0 & 1199.7 & 103.28 & 8417.2 & 1238.6 & 103.86 & 7949.30 & 1070.20 & 83.80 \\
\hline 3 & 8497.0 & 1174.4 & 103.63 & 8729.0 & 1242.1 & 106.07 & 8273.60 & 1202.90 & 78.26 \\
\hline 4 & 8264.7 & 1256.1 & 102.93 & 8268.7 & 1270.6 & 102.11 & 7969.20 & 1164.90 & 79.06 \\
\hline 5 & 8468.3 & 1256.7 & 104.17 & 8766.4 & 1304.7 & 104.19 & 7970.50 & 1270.70 & 80.15 \\
\hline 6 & 8836.9 & 1401.4 & 110.00 & 8607.8 & 1333.9 & 104.34 & 8455.70 & 1193.80 & 82.14 \\
\hline 7 & 8735.9 & 1249.2 & 106.56 & 8651.7 & 1160.1 & 105.02 & 8371.40 & 1217.20 & 79.48 \\
\hline 8 & 8530.0 & 1162.1 & 105.29 & 7780.0 & 1186.6 & 102.51 & 8460.80 & 1087.30 & 81.25 \\
\hline 9 & 7786.0 & 1282.4 & 104.34 & 9109.0 & 1105.0 & 103.94 & 8136.00 & 1226.30 & 77.17 \\
\hline 10 & 8553.7 & 1096.8 & 105.41 & 8053.6 & 1224.9 & 103.91 & 8132.80 & 1026.90 & 78.08 \\
\hline Average & 8483.6 & 1224.7 & 105.042 & 8482.9 & 1224.1 & 104.02 & 8141.48 & 1159.63 & 79.81 \\
\hline Best & 7786.0 & 1096.8 & 102.93 & 7780.0 & 1105.0 & 102.11 & 7695.50 & 1136.10 & 77.17 \\
\hline Worst & 8914.2 & 1401.4 & 110.00 & 9109.0 & 1333.9 & 106.07 & 8460.80 & 1087.30 & 83.80 \\
\hline
\end{tabular}

and running time of the three algorithms for the problem studied in this paper are showed in Table 9.

The difference between the three algorithms is that IAGA adds a reversal operation basis on adaptive genetic algorithm (AGA). The difference between HCGAVNS and the other two algorithms is that HCGAVNS uses an adaptive variable neighborhood search algorithm to replace mutation operation and uses a chaotic system to generate initial solutions to increase the diversity of the population. As can be seen from Table 9, the algorithm designed in this paper is superior to AGA and IAGA in both solution quality and efficiency. This is mainly because the chaotic systems increase the diversity of initial solutions and improve the solution quality. Adaptive variable neighborhood search algorithm uses the neighborhood structure composed of different actions to carry out an alternate search and achieves a good balance between concentration and evacuation. The adaptive search mechanism adopts the adaptive neighborhood search number strategy to control the number of neighborhood searches and improves the solution efficiency of the algorithm. 


\section{Conclusions}

This paper studies the dynamic multicompartment refrigerated vehicle routing problem with multigraph based on real-time traffic information. The main conclusions are as follows:

(1) The dynamic multicompartment refrigerated vehicle routing problem with multigraph based on real-time traffic information is a deepening and expansion of VRP. It is beneficial to improve delivery efficiency based on real-time traffic information reflected by a big data platform and to fully combine the complex and diverse characteristics of the road network environment in real life to plan delivery routes.

(2) The model considers the impact of vehicle routing planning on fresh products damage, refrigeration cost, vehicle fixed cost, and time window penalty cost on the total cost, which can more objectively and accurately reflect the operation of the cold chain logistics system.

(3) The HCGAVNS algorithm is designed to ensure the diversity of initial solutions by using chaotic phenomena, and the strategy of combining elite reservation and roulette is adopted in the selection operation to ensure the effective convergence of the algorithm. Moreover, evolutionary operation and adaptive search mechanism are used to improve the local search capability, besides the solution quality.

The research in this paper applies to the delivery network based on the real road network, which can further reduce the delivery cost of logistics enterprises and provide a theoretical solution for logistics enterprises to use traffic big data. In the future, we will consider the scenes closer to the actual delivery, such as delivery network sharing. We will also study the convergence speed of the algorithm and the further improvement of the quality of the optimal solution.

\section{Data Availability}

The data used to support the findings of this study are available at https://www.d.umn.edu/tdrl/index.htm https:// www.bernabe.dorronsoro.es/vrp/ and references [17, 34, 39, 40].

\section{Conflicts of Interest}

The authors declare that they have no conflicts of interest.

\section{Acknowledgments}

This work was supported by the Special Project of National Emergency Management System Construction of the Chinese National Funding of Social Science (20VYJ024).

\section{References}

[1] J. H. Tang, S. F. Tong, and G. C. Chen, "Vehicle routing optimization with carbon emissions considered under timevarying network," Systems Engineering, vol. 33, no. 33, pp. 37-44, 2015.
[2] X. Lin, Q. Q. Shao, Z. H. Yang, Q. Xu, and Z. H. Jin, "The Scheduling Optimization of Distribution Vehicles Considering Urban Road Network Conditions," Operation Research \& Management, vol. 28, pp. 13-23, 2019.

[3] X. L. Ge and H. Zhang, "Study on the Optimization of Vehicle Routing Problem in Urban Real Time Traffic Network," Industrial Engineering. \& Management, vol. 23, pp. 140-149+156, 2018.

[4] Z. Q. Tong and P. X. Li, "Vehicle routing problem of refined oil distribution consider real-time traffic condition and vehicle turnover rat," Industrial Engineering \& Management, vol. 24, no. 2, pp. 109-115, 2019.

[5] Y. F. Li, Z. Y. Gao, and J. Li, "Vehicle routing problem in dynamic urban network with real-time traffic information," System Engineering Theory \& Practice, vol. 33, pp. 1813-1819, 2013.

[6] A. L. Kok, E. W. Hans, and J. M. J. Schutten, "Vehicle routing under time-dependent travel times: the impact of congestion avoidance," Computers \& Operations Research, vol. 39, no. 5, pp. 910-918, 2012.

[7] N. R. Sabar, A. Bhaskar, E. Chung, and A. Turky, "A selfadaptive evolutionary algorithm for dynamic vehicle routing problems with traffic congestion," Swarm \& Evolutionar. Computer, vol. 44, pp. 1018-1027, 2019.

[8] J. X. Xu and J. N. Guo, "Research on logistics vehicle routing problem based on big data framework," Transportation System Engineering. \& Information, vol. 18, pp. 86-93, 2018.

[9] D. Q. Tang, G. G. Jin, and W. Q. Shi, "Dynamic vehicle routing schedule algorithm based on big-data platform," Computer Engineering, vol. 44, pp. 74-78, 2018.

[10] Y. G. Yao and S. Y. He, "Research on optimization of distribution route for cold chain logistics of agricultural products based on traffic big data," Management Review, vol. 31, pp. 240-253, 2019.

[11] T. Garaix, C. Artigues, D. Feill, and D. Josselin, "Vehicle routing problems with alternative paths: an application to ondemand transportation," European Journal of Operational Research, vol. 204, pp. 62-75, 2010.

[12] H. B. Ticha, N. Absi, D. Feillet, and A. Quilliot, "Multigraph modeling and adaptive large neighborhood search for the vehicle routing problem with time window," Computer Operational Research, vol. 104, pp. 113-126, 2019.

[13] H. W. Wang, YenYi Lee, and Y. Y. Lee, "Two-stage particle swarm optimization algorithm for the time dependent alternative vehicle routing problem," Journal of Applied \& Computational Mathematics, vol. 03, no. 04, pp. 1-9, 2014.

[14] Y. X. Huang, L. Zhao, T. V. Woensel, and J. P. Gross, "Timedependent vehicle routing problem with path flexibility," Transportation Research Part B: Methodological, vol. 95, pp. 169-195, 2017.

[15] M. Setak, M. Habibi, H. Karim, and M. Abedzadeh, "A timedependent vehicle routing problem in multigraph with FIFO property," Journal of Manufacturing Systems, vol. 35, pp. 37-45, 2015.

[16] J. N. Qian and R. Eglese, "Eglese Fuel emissions optimization in vehicle routing problems with time-varying speeds," $E u$ ropean Journal of Operational Research, vol. 248, pp. 840-848, 2016.

[17] S. Y. Li, B. Dan, and X. L Ge, "Optimization model and algorithm of low carbon vehicle routing problem under multigraph time-varying network," Computer Integrated Manufacturing Systems, vol. 25, pp. 454-468, 2019.

[18] J. M. Chen, N. Zhou, and Y. Wang, "Optimization of multicompartment cold chain distribution vehicle routing for fresh 
agricultural products," Systems Engineering, vol. 36, pp. 106-113, 2018.

[19] C. L. Wang and S. W. Li, "A multi-compartment vehicle distribution route optimization method based on complex network," Journal of Systems Management, vol. 28, pp. 708716, 2019.

[20] S. Martins, M. Ostermeier, P. Amorim, A. Hübner, and B. Almada-Lobo, "Product-oriented time window assignment for a multi-compartment vehicle routing problem," European Journal of Operational Research, vol. 276, pp. 893-909, 2019.

[21] R. Eshtehadi, E. Demir, and Y. Huang, "Solving the vehicle routing problem with multi-compartment vehicles for city logistics," Computer Operational Research, vol. 115, Article ID 104859, 2020.

[22] I. Kaabachi, H. Yahyaoui, S. Krichen, and A. Dekdouk, "Measuring and evaluating hybrid metaheuristics for solving the multi-compartment vehicle routing problem," Measurement, vol. 141, pp. 407-419, 2019.

[23] L. Chen, Y. Liu, and A. Langevin, "A multi-compartment vehicle routing problem in cold-chain distribution," Computer Operational Research, vol. 111, pp. 58-66, 2019.

[24] M. Ostermeier and A. Hübner, "Vehicle selection for a multicompartment vehicle routing problem," European Journal of Operational Research, vol. 269, pp. 682-694, 2018.

[25] W. Hu, C. J. Liang, and L. B. Fan, "Optimization of multitemperature joint-delivery based on simultaneous pickup and delivery," Journal Guangxi University, vol. 41, pp. 1576-1584, 2016.

[26] T. Henke, M. Grazia Speranza, and G. Wäscher, "The multicompartment vehicle routing problem with flexible compartment sizes," European Journal of Operational Research, vol. 246, pp. 730-743, 2015.

[27] J. C. Goodson, "A priori policy evaluation and cyclic-orderbased simulated annealing for the multi-compartment vehicle routing problem with stochastic demands," European Journal of Operational Research, vol. 241, no. 2, pp. 361-369, 2015.

[28] M. Alinaghian and N. Shokouhi, "Multi-depot multi-compartment vehicle routing problem solved by a hybrid adaptive large neighborhood search," Omega, vol. 76, pp. 85-99, 2018.

[29] L. C. Coelho and G. Leandro, "Laporte. Classification models and exact algorithms for multi-compartment delivery problems," European Journal of Operational Research, vol. 242, pp. 854-864, 2015.

[30] A. Osvald and L. Z. Stirn, "A vehicle routing algorithm for the distribution of fresh vegetables and similar perishable food," Journal of Food Engineering, vol. 85, pp. 285-295, 2008.

[31] X. H. Miao, X. X. Zhou, S. Lin et al., "Study on routing optimization for cold-chain logistics distribution of 3PL," Operational Research \& Management, vol. 20, pp. 32-38, 2011.

[32] M. Zhang and J. H. Qu, "Dispatching network optimization for cold chain of perishable products based on improved genetic algorithm," Logistics Engineering \& Management, vol. 37, pp. 139-142, 2015.

[33] C. Du and Y. J. Li, "Research on Cold Chain Distribution Routing Problem Based on Customer Satisfaction and Minimum Loss," Industrial Engineering \& Management, vol. 25, no. 6, pp. 163-171, 2020.

[34] W. T. Fang, Z. Z. Ai, Q. Wang, and J. B. Fan, "Research on cold chain logistics distribution path optimization based on hybrid ant colony algorithm," China Management Science, vol. 27, pp. 107-115, 2019.

[35] D. H. Tao, R. Liu, Y. J. Lei, and Q. X. Zhang, "A research on routing optimization of cold chain distribution based on green supply chain," Indurial Engineering, vol. 22, pp. 89-95, 2019.

[36] Z. X. Zhao, X. M. Li, X. C. Zhou, and C. S. Liu, "Research on green vehicle routing problem of cold chain distribution: considering traffic congestion," Computer Engineering \& Applied, vol. 56, pp. 224-231, 2020.

[37] S. M. Xu, "Study on vehicle routing problem with cold chain logistics with time windows," Logistics Science \& Technology, vol. 40, pp. 80-84, 2017.

[38] D. Hou, H. Fan, X. Ren et al., "Time-dependent multi-depot heterogeneous vehicle routing problem considering temporal-spatial distance," Sustainability, vol. 13, no. 9, p. 4674, 2021.

[39] C. J. Liang and Q. Q. Zou, "Research on vehicle routing problem with temperature variable in cold chain logistics," Journal Guangxi University, vol. 42, pp. 1802-1809, 2017.

[40] M. Reed, A. Evering, and E. Roxanne, "An ant colony algorithm for the multi-compartment vehicle routing problem," Applied Soft Computing, vol. 15, pp. 169-176, 2014. 